\title{
Curriculum Radiologie: Ein lebendiges Dokument, das zum Austausch einlädt
}

Warum ein eigenes Curriculum? Professor Birgit Ertl-Wagner von der LMU München definiert die Relevanz dieses radiologischen Lernzielkatalogs für die medizinische Lehre.

\section{Frau Professor Ertl-Wagner, weshalb gibt es jetzt überhaupt ein Curricu- lum für die Radiologie?}

Es ist für die Radiologie als Fachgebiet wichtig, bei der curricularen Planung an den medizinischen Fakultäten beteiligt zu sein. Nur so kann gewährleistet werden, dass die Medizinstudierenden die Radiologie in ihrer ganzen Breite und Vielfalt kennenlernen. Das Curriculum soll den Kolleginnen und Kollegen dabei als Leitfaden dienen. In seinem modularen Aufbau kann es an die individuellen Gegebenheiten vor Ort angepasst werden. Wir haben außerdem innerhalb der Deutschen Röntgengesellschaft und gemeinsam mit anderen Strahlenfächern in den Jahren 2014 und 2015 den von der Gesellschaft für medizinische Ausbildung (GMA) und dem Medizinischen Fakultätentag e.V. (MFT) erstellten Nationalen Kompetenzbasierten Lernzielkatalog Medizin (NKLM) diskutiert. Wir kamen dabei zum Ergebnis, dass die Radiologie als eigenes Fach im NKLM nicht deutlich genug präsentiert und daher nicht genügend wahrgenom- men wird. Das gab den Anstoß für ein eigenes starkes Curriculum Radiologie.

\section{Wieso ist die Radiologie - im NKLM} oder auch im Medizinstudium selbst - unterrepräsentiert?

Die Radiologie wird gerne als „Querschnittfach" bezeichnet, da sie nahezu alle Bereiche der Medizin tangiert. Da ist die Gefahr nicht zu unterschätzen, dass sie in einer organsystembasierten Lehre von den anderen Fächern quasi vereinnahmt wird. Das wollen wir verhindern.

\section{Worauf begründet sich Ihre Hoff-} nung, dass sich dies künftig ändern wird?

Der springende Punkt ist meines Erachtens die Präsenz der Kolleginnen und Kollegen vor Ort - sowohl in der curricularen Planung als auch in der Lehre selbst. Im Englischen gibt es den Ausdruck: „If you are not at the table, you will be on the menu". Und wer will schon auf der Spei-

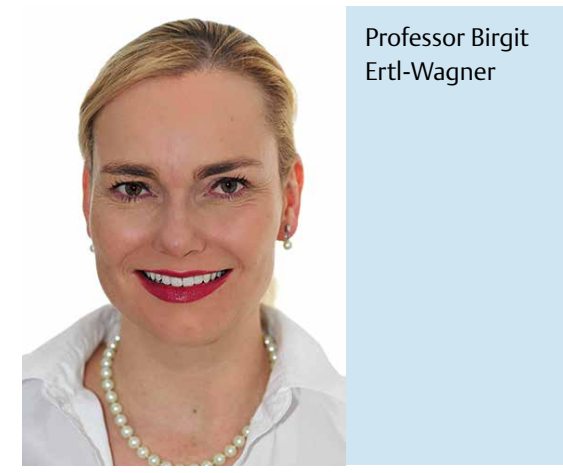

sekarte landen? Das Curriculum soll eine Hilfe darstellen, indem es eine Art radiologischen Lernzielkatalog darstellt, der an die lokalen Gegebenheiten angepasst werden kann.

\section{Was passiert nun mit dem Curriculum?}

Die Deutsche Röntgengesellschaft verbreitet das Curriculum über ein Mailing an alle ihre Mitglieder. Außerdem ist das Curriculum auf der Webseite der DRG abrufbar und wird in der RöFo veröffentlicht. Es soll als lebendiges Dokument verstanden werden - Änderungsvorschläge oder Ergänzungen sind jederzeit willkommen.

Vielen Dank für das Gespräch! 